\title{
Decisions Relating to Cardiopulmonary Resuscitation: commentary 1: CPR and the cost of autonomy
}

Robin Gill University of Kent at Canterbury

Since the last generation medical ethics has seen a remarkable shift from benign medical paternalism to patient rights and autonomy. Whereas once it might have been acceptable for doctors to decide, largely on their own, what was in the best interests of their patients, today senior health professionals are expected to make decisions jointly both with patients or their carers and with other health professionals. Patient autonomy and justice, and not simply beneficence, are usually thought to be crucial to medical ethics today.

Although I strongly support this shift in medical ethics, I believe it is important to recognise that it has some cost to medical professionals, to patients and even at times to the National Health Service (NHS). Medical paternalism, when it was genuinely benign, did have some benefits both for doctors and for their patients. Yet precisely because most of us are no longer prepared to be at the receiving end of such paternalism, these particular benefits are now largely lost to us.

The new joint statement, Decisions Relating to Cardiopulmonary Resuscitation, ${ }^{1}$ illustrates this point well. At the outset it admits that "health professionals are aware that decisions about attempting resuscitation raise very sensitive and potentially distressing issues for the patient and people emotionally close to the patient". It was because of these sensitive and potentially distressing issues that it was once thought acceptable to make do not attempt resuscitation (DNAR) (or DNR as it was then) orders without consulting either patients or their families and with the knowledge that the latter would probably never discover that such orders had ever been made. Now, however, health workers are warned frankly that they "should remember that patients are legally entitled to see and have a copy of their health records". As in many other areas of life, an individual's right to the disclosure of information held about her has breached areas of even well-intentioned public secrecy. Today DNAR orders made in secret, even those orders made to protect vulnerable patients, are no longer acceptable. Indeed, media attention in this area was initially driven by competent adults discovering on their discharge from hospital that they had been subject, without their knowledge, to DNAR orders.

Yet there is a cost here both to patients and to health professionals. The joint statement admits that "discussions of the advisability or otherwise of attempting CPR are highly sensitive and complex and should be undertaken by senior, experienced members of the medical team who have received appropriate training. Support should come from similarly trained senior nursing colleagues". So, once again, it is expected that it is senior doctors and nurses who must give time and attention (and be trained) to discuss CPR with their vulnerable patients. This, in turn, must be set alongside their other duties to inform all of their patients properly about the potential risks and benefits of different forms of treatment and to reach an agreed "concordance" about their often complex medication. Whereas in the past they might have relied upon more junior colleagues to impart such information (if such information was properly imparted at all), now it is senior health professionals who must be thoroughly involved. Naturally this also has implications for NHS resources: if senior health professionals are to invest their time in this way, then it is likely that they will have less time for performing operations or whatever. Then, presumably, unless they are to work even longer hours, either waiting lists will become longer, or more senior health professionals will be required in the NHS.

There is also a cost to the patients themselves, as the joint statement again clearly recognises:

"People have ethical and legal rights to be involved in decisions that relate to them. Because patients' own views about the level of burden or risk they consider acceptable carry considerable weight in deciding whether treatment is given, it follows that decisions about whether the likely benefits from successful CPR outweigh the burdens should be discussed with competent patients. Thus where competent patients are at foreseeable risk of cardiopulmonary arrest, or have a terminal illness, there should be sensitive exploration of their wishes regarding resuscitation .... Competent patients should understand that there are opportunities to 
318 Clinical ethics: Decisions relating to cardiopulmonary resuscitation: commentary 1: CPR and the cost of autonomy

talk about attempting CPR, but should not be forced to discuss the issue if they do not want to."

Some patients may well be distressed by any such exploration, however tentative and sensitive. The very fact that the issue of resuscitation is being discussed will clearly indicate to the competent that DNAR is at least being contemplated. For many of us this may not be a problem. Perhaps we have already come to terms with our finitude, perhaps we are sanguine about the prospect of annihilation, or perhaps we hope or believe that this life is not the end at all. Yet there is abundant evidence in philosophical, psychological and spiritual classics that, whatever our different eschatological convictions, angst about death is widespread among human beings.

Discussions about CPR with vulnerable patients may well involve such angst. Not the least of the problems here, as the joint statement recognises, is that media portrayals tend to encourage many patients to overestimate the success rate and underestimate the invasiveness of CPR. Yet, disturbingly, repeated attempts at CPR can themselves be deeply demoralising both for the patients and for the health professionals involved.

Of course the troubled patient can simply refuse to discuss CPR and, in effect, opt for medical paternalism instead. Here the joint statement notes that "where a DNAR order is made and there has been no discussion with the patient because he or she has indicated a clear desire to avoid such discussion, this must be documented in the health records and the reasons given. As with any other aspect of care, health professionals must be able to justify their actions". It is the words "clear desire" and "must be able to justify" that indicate the tension here. Precisely because health professionals have been criticised in the past for making DNAR orders without consulting competent patients, it is crucial that they are able to justify any DNAR orders which are made now without the explicit and informed consent of the patients involved. Yet if patients are unwilling to be involved in such explicit discussions and, in effect, delegate the decision to the health professionals, then it is important that the latter (if only for their own protection) are able to demonstrate later that this was a "clear desire" of those patients. Yet just how are they to establish this "clear desire" without these very patients being involved in a discussion about at least the initial stages of the subject (death and CPR) which causes them such angst?

Here is the cost of autonomy. A right which many of us cherish-namely a right for the competent to be properly informed before treatment is given or withdrawn/withheld-may become a burden for others when they are at their most vulnerable. It is not simply that properly informed consent may involve the attempt by people without any background or training to understand information that is complex and confusing. There are in any case many areas of modern medicine, especially those emerging as the result of developing biotechnology, that are far more complex and confusing. Nor is it simply a matter of finding the appropriate medium for imparting information relevant to properly informed consent (the joint statement mentions the importance of trusts, hospitals, general practices and residential care facilities all providing appropriate written information about their resuscitation policies). It is more than that. In whatever way the information about CPR is conveyed to vulnerable patients who are feeling understandable angst about death, some may well be deeply troubled about even the most sensitive, preliminary and tentative discussion of DNAR. How could it be otherwise?

The old paternalism was undoubtedly comforting for many in an age of greater deference to relatively elite professional people. Now, at a time when a third of young people enter tertiary education, and when middle-class professionals have become increasingly dominant, such paternalism appears anachronistic. We expect to be properly informed about anything that affects our lives, including health care, and even about CPR. This is right, but it also has a cost.

Robin Gill is Michael Ramsey Professor of Modern Theology, University of Kent at Canterbury.

\section{References}

1 British Medical Association, Resuscitation Council (UK) and the Royal College of Nursing. Decisions relating to cardiopulmonary resuscitation: a joint statement from the British Medimonary resuscitation: a joint statement from the British Medical Association, the Resuscitation Council (UK) and the Royal 2001. Fournal of Medical Ethics 2001;27:312-18. 